\title{
Cluster radioactivity in superheavy nuclei
}

\author{
M. Warda, ${ }^{1, *}$ A. Zdeb, ${ }^{1,2}$ and L. M. Robledo ${ }^{2}$ \\ ${ }^{1}$ Katedra Fizyki Teoretycznej, Uniwersytet Marii Curie-Sktodowskiej, 20031 Lublin, Poland \\ ${ }^{2}$ Departamento de Física Teórica, Universidad Autónoma de Madrid, 28049 Madrid, Spain
}

(Received 29 June 2018; published 24 October 2018)

\begin{abstract}
Cluster radioactivity is an exotic nuclear decay observed in actinides where a light nucleus is emitted while the remaining heavy mass residue is the doubly magic ${ }^{208} \mathrm{~Pb}$ or a nucleus in its neighborhood. We have investigated this type of decay in heavier nuclei up to $\operatorname{Lv}(Z=116)$ within a microscopic theory. It has been found that the cluster radioactivity known in the light actinides may become the dominant decay channel in some superheavy nuclei. This superasymmetric fission channel is distinct from typical asymmetric fission in actinides. We predict a sharp fission fragment mass distribution with the heavy fragment close to ${ }^{208} \mathrm{~Pb}$.
\end{abstract}

DOI: 10.1103/PhysRevC.98.041602

Introduction. In a seminal experiment carried out by Rose and Jones in 1984 [1] a new type of nuclear decay was discovered. Immersed in an enormous $\alpha$ decay background produced by the parent nucleus ${ }^{223} \mathrm{Ra}$ a few events producing ${ }^{14} \mathrm{C}$ were observed. The phenomenon received the name of cluster radioactivity (CR) due to the intermediate mass of the light fragment emitted. In the following years, 20 other cluster emitters were discovered, see reviews in Refs. [2,3]. In this type of decay, light nuclei ranging from ${ }^{12} \mathrm{C}$ to ${ }^{34} \mathrm{Si}$ are emitted by actinides from ${ }^{221} \mathrm{Fr}$ to ${ }^{242} \mathrm{Cm}$. The remaining heavy mass fragment in all these reactions is either the doubly magic ${ }^{208} \mathrm{~Pb}$ or one of its neighbors. The double magic structure of the heavy fragment clearly shows the strong influence of shell effects on CR. This is an exotic process: typical branching ratios to the dominant $\alpha$ emission are as low as $10^{-6}-10^{-12}$ and consequently the half-lives range from $10^{15}$ to $10^{25} \mathrm{~s}$. For nuclei heavier than ${ }^{242} \mathrm{Cm}$ spontaneous fission becomes a competing channel increasing the difficulty to detect $\mathrm{CR}$ products among the numerous fission fragments present in the background. As a consequence, no $\mathrm{CR}$ has been observed in nuclei around mass $A=250$.

Cluster radioactivity is usually described in the spirit of the Gamow model of $\alpha$ decay, assuming that a preformed cluster tunnels through a potential barrier [4-7]. The exponential dependence of the tunneling probability with the parameters of the barrier leads to a modified Geiger-Nuttall law [8-11] relating half-lives for CR to the $Q$ value of the reaction. This approach presents two main disadvantages: first, it requires a model to estimate the preformation probability of the cluster; and second, a local fit of additional model parameters is required to reproduce observed decay half-lives. An alternative to this model is to treat $\mathrm{CR}$ as a very asymmetric fission process to be described with the tools of the traditional fission decay model. This was the approach followed in Ref. [12] to predict the existence of CR a few years before its experimental discovery. The most important ingredient of any fission model

*michal.warda@umcs.pl is the analysis of the changes in energy of the nucleus as it changes deformation in its way to scission $[13,14]$. In the context of CR, it was shown [15] that a superasymmetric fission valley can be found on the potential energy surface (PES) spanned by the quadrupole and octupole moments. It leads to a scission point with ${ }^{208} \mathrm{~Pb}$ or a nucleus of a very similar mass as one of the fission fragments. In the light actinides the fission barrier associated with this channel reaches a height of $25 \mathrm{MeV}$, which is the right order of magnitude to reproduce CR half-lives.

Several authors have already suggested that superheavy (SH) elements may decay through CR [16-19]. Calculations performed within phenomenological approaches and semiempirical formulas show a trend to predict shorter half-lives for $\mathrm{CR}$ in the $\mathrm{SH}$ region. Therefore, the competition with other disintegration channels becomes relevant and might have an impact on the very limits of the periodic table [20] or in the $r$-process nucleosynthesis [21].

The aim of this work is to study the possible existence of $\mathrm{CR}$ in $\mathrm{SH}$ nuclei within a fully microscopic theory. To this end we have used the self-consistent Hartree-Fock-Bogoliubov model with the Gogny D1S interaction to calculate the PES and the collective inertias required for the evaluation of $\mathrm{CR}$ half-lives. As relevant collective coordinates, the axially symmetric quadrupole and octupole moments are used. This is a well-established quantum mechanics approach that allows us to describe a very rich variety of nuclear shapes not limited by the number of deformation parameters. This type of calculation has been successfully applied to the description of both CR [22] and fission in heavy [23] and SH nuclei [24,25].

One of the common features of fission and CR is that the $Z$ and $N$ values of their fragments approximately conserve the $N / Z$ ratio of the parent nucleus. Therefore, to investigate the possibility to observe $\mathrm{CR}$ in heavier nuclei, one has to focus on possible emitters with the $N / Z$ value of $126 / 82=1.537$ corresponding to the always present heavy fragment of ${ }^{208} \mathrm{~Pb}$. Therefore, we have chosen for our studies a set of even-even isotopes, one for each element, with an $N / Z$ ratio close to 1.537 , namely, ${ }^{224} \mathrm{Ra},{ }^{228} \mathrm{Th},{ }^{234} \mathrm{U}$, and ${ }^{238} \mathrm{Pu}$, where $\mathrm{CR}$ 


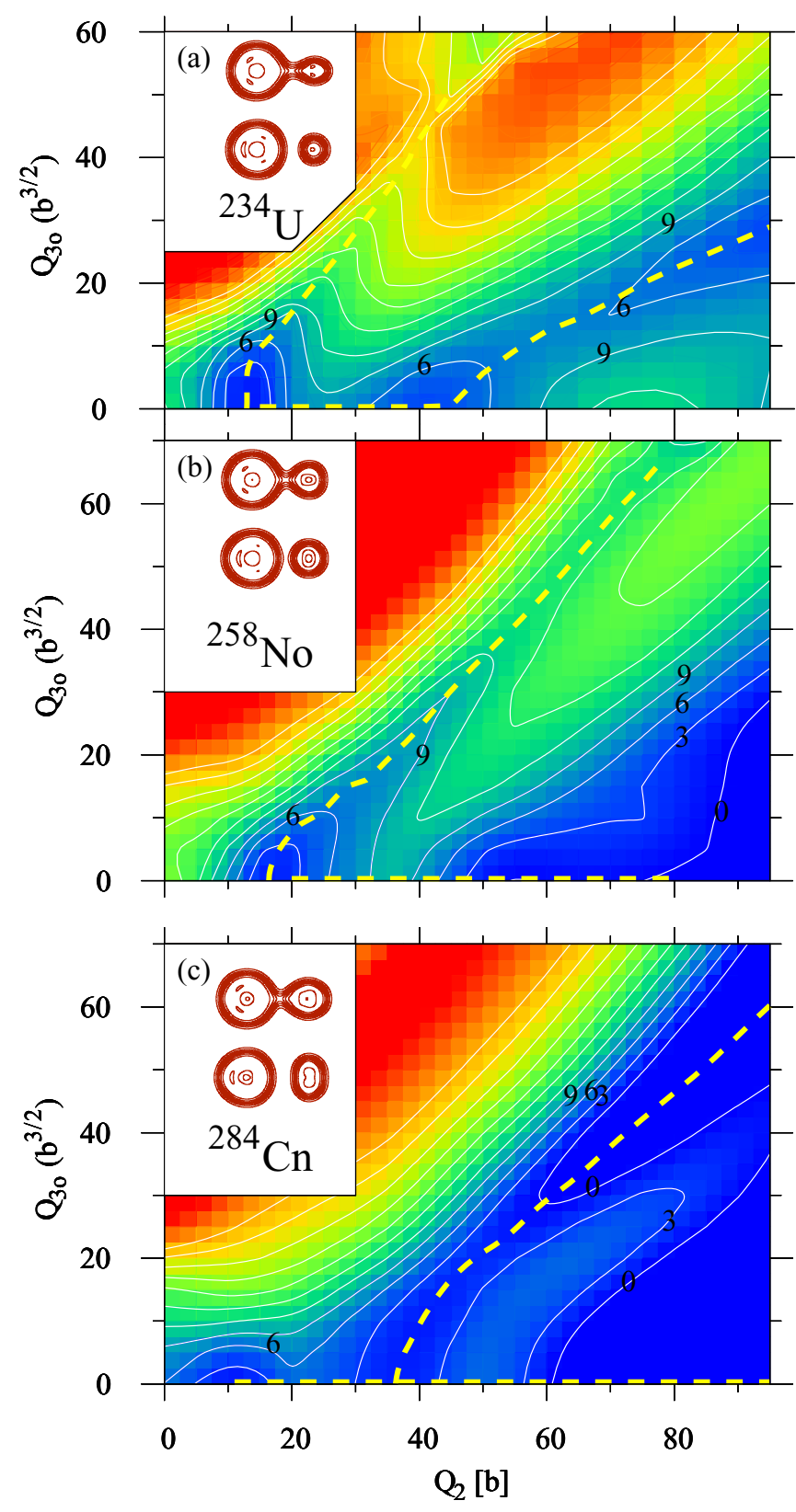

FIG. 1. PES of (a) ${ }^{234} \mathrm{U}$, (b) ${ }^{258} \mathrm{No}$, and (c) ${ }^{284} \mathrm{Cn}$. Constant energy lines are plotted every $3 \mathrm{MeV}$. Fission paths are marked with yellow dashed lines. Insets show pre- and post-scission configurations.

has already been observed. Heavier isotopes include ${ }^{244} \mathrm{Cm}$, ${ }^{248} \mathrm{Cf},{ }^{254} \mathrm{Fm},{ }^{258} \mathrm{No},{ }^{264} \mathrm{Rf},{ }^{268} \mathrm{Sg},{ }^{274} \mathrm{Hs},{ }^{278} \mathrm{Ds},{ }^{284} \mathrm{Cn},{ }^{290} \mathrm{Fl}$, and ${ }^{294} \mathrm{Lv}$. The final part of this chain belongs to the region of SH elements close to the isotopes experimentally produced in hot fusion reactions [26]. ${ }^{284} \mathrm{Cn}$ is the heaviest isotope that has been observed decaying through fission both at GSI and in Dubna $[27,28]$. These are difficult experiments due to the low production rate and a total number of only 28 fission events have been reported for this isotope.

Results. A typical example of a nucleus where CR has been observed is the light actinide ${ }^{234} \mathrm{U}$. In the PES of this nucleus, shown in Fig. 1(a), the asymmetric fission path, characteristic for heavy nuclei up to Fm is easily found. It goes from the ground state through the first symmetric fission barrier $(6.6 \mathrm{MeV})$. Then it crosses the second asymmetric saddle and gets to the scission point. On the same PES another valley can be noticed. It begins at the ground state and heads directly toward octupole deformed shapes. Along most of the whole fission path in this valley both quadrupole and octupole moments increase simultaneously up to $Q_{20}=45 \mathrm{~b}$ and $Q_{30}=51 \mathrm{~b}^{3 / 2}$. In the saddle point, where the scission point is located, the energy reaches $26.6 \mathrm{MeV}$. From there on, the PES corresponds to the Coulomb energy of the two fragments as they drift away and the energy decreases hyperbolically with the increasing distance between the fragments. The nuclear matter density distributions before and after scission are plotted in the inset of Fig. 1(a). It is easy to find that the mass and shape of the heavy fragment correspond to the spherical doubly magic lead isotope. The two fragments are well defined far before the rupture of the neck. The fragment matter distribution proves that this superasymmetric fission channel describes CR. The same topology of the PES has been also found in all cluster emitters observed in the light actinides [15]. For details of the calculation, see Refs. [15,24].

The CR valley in ${ }^{258}$ No depicted in Fig. 1(b) shows the same characteristics as in ${ }^{234} U$ but it is shifted toward larger quadrupole moments. The scission point is located at higher quadrupole and octuple deformation, making the fission barrier broader. The height of the CR fission barrier is reduced to $14.2 \mathrm{MeV}$. Again, the mass of a heavy fragment at scission corresponds to $A=208$. In the superheavy ${ }^{284} \mathrm{Cn}$ [Fig. 1(c)] the same type of very asymmetric fission valley, shifted toward higher $Q_{30}$, can be found as well. In this nucleus the traditional symmetric fission barrier has a twohumped structure and the CR valley starts at the minimum located between them instead of the ground state. Moreover, the CR fission barrier has substantially changed its shape: it is much lower than in previous cases, reaching a height of only $1.5 \mathrm{MeV}$ at the saddle point. Starting from $Q_{20}=60 \mathrm{~b}$ this fission path drops down below the ground-state energy. The scission point is located at $Q_{20}=128 \mathrm{~b}$ and $Q_{30}=92 \mathrm{~b}^{3 / 2}$ with an energy $12.6 \mathrm{MeV}$ below ground state.

To study the properties of $\mathrm{CR}$ in the $\mathrm{SH}$ region we have computed PESs for all aforementioned isotopes and we have found that the CR valley exists in all considered nuclei. In Fig. 2(a) a bunch of CR fission paths is shown. Its characteristic pattern present in the PES smoothly evolves in going from light actinides to $\mathrm{SH}$ nuclei. The only sharp modification takes place in the starting point of the paths for ${ }^{268} \mathrm{Sg}$ and heavier nuclei. They start at the second minimum, not at the ground state, as it was described above in the case of ${ }^{284} \mathrm{Cn}$. In these isotopes the CR fission barrier is comprised of two differentiated parts: first a reflection symmetric hump followed by a second octupole deformed barrier. In all considered isotopes the CR scission configuration contains a spherical heavy mass fragment: the doubly magic ${ }^{208} \mathrm{~Pb}$. This means that in $\mathrm{SH}$ elements one may expect decay of the same nature as in light actinides.

A similar structure of fission paths in this region was also found with the covariant density theory [31] and Skyrme energy density functional [32] in a nonrelativistic setup. The 


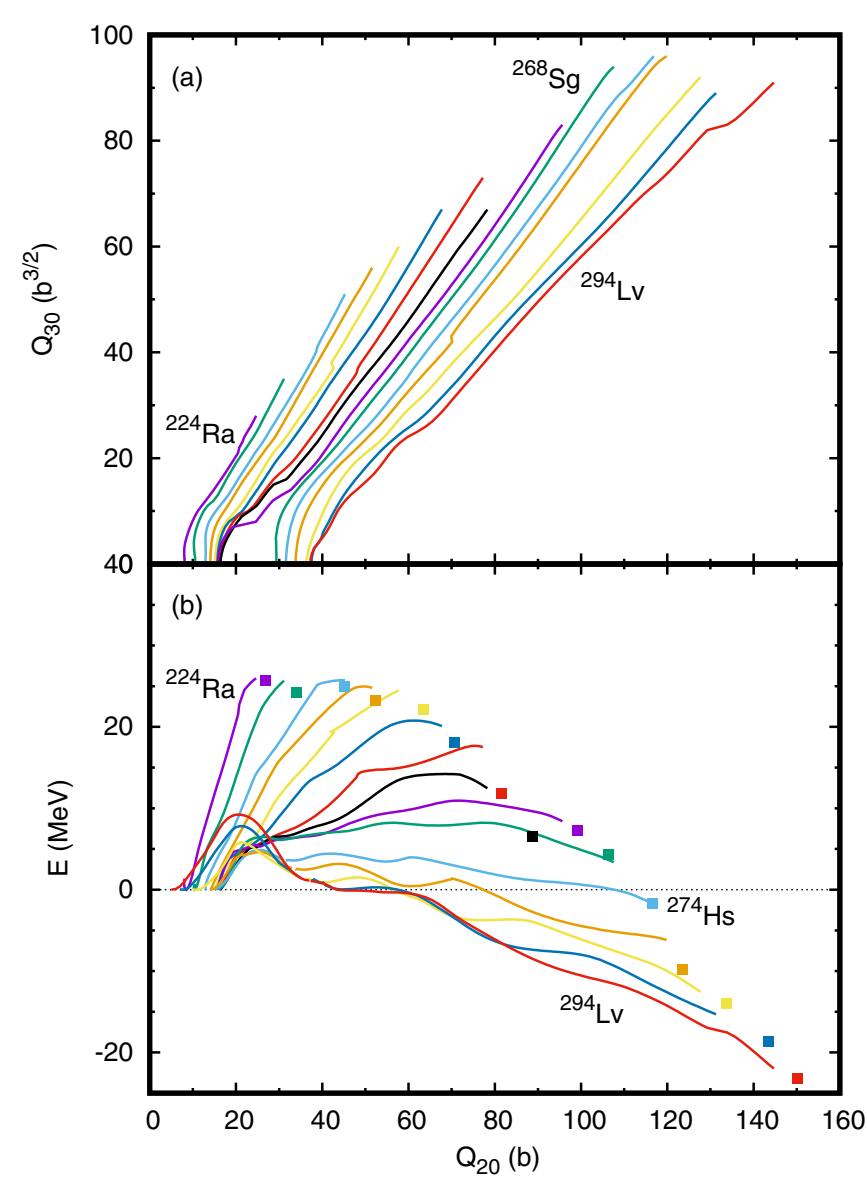

FIG. 2. (a) CR fission paths in quadrupole and octupole coordinates for isotopes: ${ }^{224} \mathrm{Ra},{ }^{228} \mathrm{Th},{ }^{234} \mathrm{U},{ }^{238} \mathrm{Pu},{ }^{244} \mathrm{Cm},{ }^{248} \mathrm{Cf},{ }^{254} \mathrm{Fm}$, ${ }^{258} \mathrm{No},{ }^{264} \mathrm{Rf},{ }^{268} \mathrm{Sg},{ }^{274} \mathrm{Hs},{ }^{278} \mathrm{Ds},{ }^{284} \mathrm{Cn},{ }^{290} \mathrm{Fl}$, and ${ }^{294} \mathrm{Lv}$. (b) Fission barriers of aforementioned nuclei. Squares correspond to scission configuration calculated with Eq. (1) with $Q$ values obtained from experimental masses [29], wherever possible, and liquid drop systematics [30].

asymmetric fragment mass distribution predicted here is not in contradiction with symmetric fission barriers obtained within macroscopic-microscopic models [33-35]. The first saddle point is reflection symmetric and much higher than the octupole deformed second one.

The height and shape of fission barriers in superasymmetric fission channels [presented in Fig. 2(b)] are the crucial features for the understanding of $\mathrm{CR}$ in heavier nuclei. In the first five isotopes they have similar heights of around $25 \mathrm{MeV}$. The scission point is gradually shifted toward higher quadrupole moments, due to the increasing size of the cluster in this configuration. Starting from ${ }^{248} \mathrm{Cf}$, the energy of the scission point gradually decreases. In the mass region between 250 and 270 the fission barriers are still very high $(8-20 \mathrm{MeV})$ and very broad. Tunneling probability across them is too low to make it possible to observe the CR channel. The situation changes substantially already in ${ }^{274} \mathrm{Hs}$. The scission point is below the ground-state energy and the second, asymmetric, fission barrier is lower than $5 \mathrm{MeV}$, which is less than the energy of the first symmetric saddle point. In ${ }^{278} \mathrm{Ds}$ and

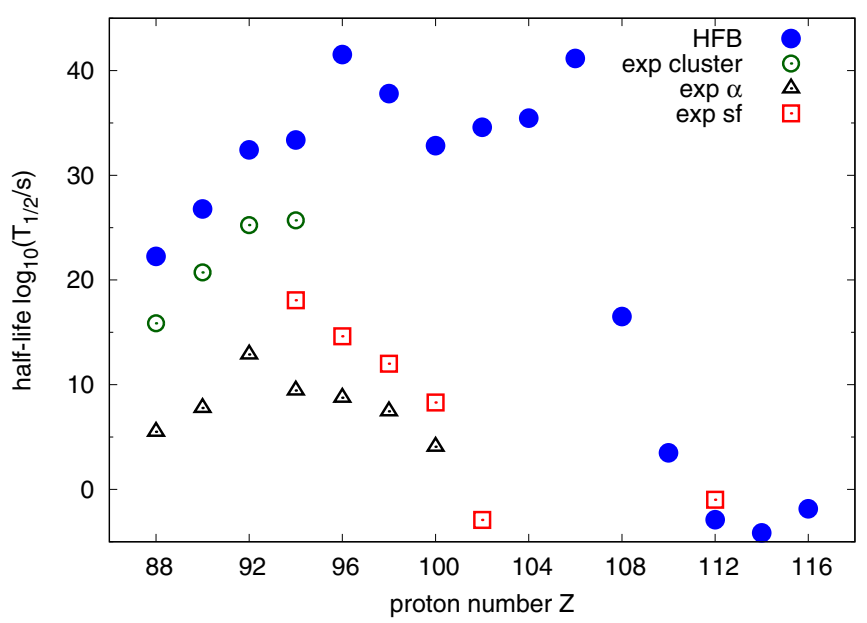

FIG. 3. CR half-lives of considered isotopes compared with experimental data of $\mathrm{CR}, \alpha$ emission, and spontaneous fission [36].

heavier nuclei almost the whole asymmetric part of the fission path is below the ground-state energy. In this way, the fission barrier consists basically of the first, symmetric, hump which is relatively narrow and easy to tunnel. The asymmetric part of the fission barrier has little influence on the half-lives but it is crucial for the asymmetry of the fragment mass distribution.

The reduction of the CR fission barrier height in the region of SH elements came as a surprise. However, it can be easily explained on the basis of a simple analysis of the Coulomb repulsion energy of two charged spheres at the scission point. The scission configuration consists of a spherical ${ }^{208} \mathrm{~Pb}$ heavy fragment and a lighter cluster created from the remaining nucleons of the parent nucleus. The separation of the fragments is given by the sum of their radii increased by a tip distance $d$. This extra spacing between daughter nuclei comes from the neck connecting pre-fragments before scission. After the neck rupture, the distances between the position of half-density of the fragments in the post-scission configuration vary from 2.5 to $4.7 \mathrm{fm}$. The energy of the scission configuration can be estimated as the Coulomb energy of two point charges minus the $Q$ value of the decay:

$$
E=k \frac{82(Z-82) e^{2}}{r_{208}+r_{A-208}+d}-Q .
$$

Here $Z$ and $A$ are the charge and mass number of the parent nucleus and the fragment's radius is estimated using the traditional $r_{A_{F}}=1.2 A_{F}^{1 / 3} \mathrm{fm}$ expression. The results obtained for the considered nuclei with an average constant tip distance value $d=3.3 \mathrm{fm}$ are plotted in Fig. 2(b) with squares. The obtained values perfectly reproduce the trend of pre-scission energies and configurations calculated within the microscopic theory, despite that the deformation of the fragments has not been taken into account and the value of $d$ is just estimated.

The reduction of the $\mathrm{CR}$ fission barrier height in the SH region affects the half-lives plotted in Fig. 3, calculated using the standard WKB approximation. The effective inertia and zero point energy in the $Q_{20}-Q_{30}$ collective space are calculated using the perturbative cranking approximation for pre-scission configuration. After scission, the reduced mass 


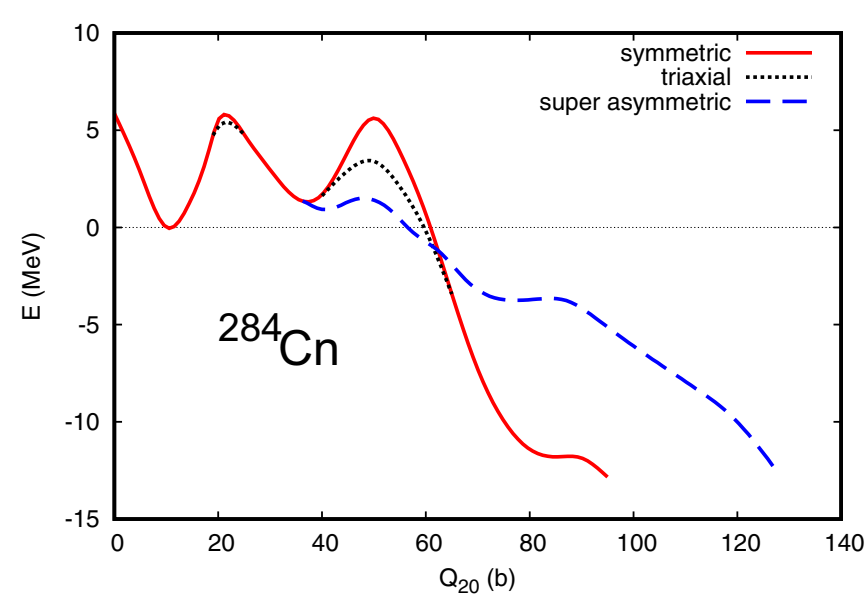

FIG. 4. Fission barrier of ${ }^{284} \mathrm{Cn}$ as a function of quadrupole moment.

is taken and a constant zero point energy is used. Half-lives longer than $10^{30} \mathrm{~s}$ are obtained in the $Z=96-106$ region. However, starting at ${ }^{274} \mathrm{Hs}$ we observe their substantial reduction as a consequence of the diminution of the superasymmetric fission barrier height [cf. Fig. 2(b)]. In nuclei with $A>280$ we obtain half-lives in the range of what could be measured in contemporary $\mathrm{SH}$ nuclei experiments. As the calculated half-life for ${ }^{284} \mathrm{Cn}$ is comparable to experimental data for fission, we will present a more detailed study of this representative isotope.

In the previous paper [24] the main decay channel of ${ }^{284} \mathrm{Cn}$ was claimed to be asymmetric fission. Here we have shown that this is instead a superasymmetric fission mode directly connected with the standard CR observed in the actinides. The other decay channels are less favored. Predictions of $\alpha$ radioactivity give 4 orders of magnitude longer half-lives. Symmetric fission in ${ }^{284} \mathrm{Cn}$ is also highly suppressed [see Figs. 1(c) and 4]. The second symmetric barrier has the same width as the asymmetric one but its height is $5.6 \mathrm{MeV}$, to be compared with $1.5 \mathrm{MeV}$ for the asymmetric one. Nonaxial deformation may reduce the height of the fission barrier of $\mathrm{SH}$ nuclei but it does not affect the main conclusion. Including the triaxial degree of freedom leaves the first fission barrier practically unchanged while the second symmetric barrier is reduced by $2.2 \mathrm{MeV}$ with $\gamma \approx 6^{\circ}$. However, this effect is too weak to make the symmetric fission channel the most favorable. The same conclusion applies to all the isotopes in the region $Z=110-114$ and $N=170-176$ centered around ${ }^{284} \mathrm{Cn}$ that were previously identified as nuclei with asymmetric fission $[24,25]$. All those nuclei should decay in the same mode as ${ }^{284} \mathrm{Cn}$. The CR fission valley also exists in heavier elements, but decay through this channel is suppressed by $\alpha$ emission. On the other hand, in lighter systems the symmetric fission mode is dominant. We may conclude that superasymmetric fission, closely related to CR in the actinides, can be found in some SH nuclei as the dominant decay mode.

Conclusions. We have shown that superasymmetric fission in $\mathrm{SH}$ nuclei has the same nature as $\mathrm{CR}$ in light actinides. The dominant decay channel of isotopes around ${ }^{284} \mathrm{Cn}$ is superasymmetric fission with doubly magic ${ }^{208} \mathrm{~Pb}$ as the heavy mass fragment. Lighter fragments corresponding to the $\mathrm{SH}$ nuclei discussed here would be ${ }^{70} \mathrm{Ni},{ }^{76} \mathrm{Zn},{ }^{82} \mathrm{Ge}$, and ${ }^{86} \mathrm{Se}$ in the fission of ${ }^{278} \mathrm{Ds},{ }^{284} \mathrm{Cn},{ }^{290} \mathrm{Fl}$, and ${ }^{294} \mathrm{Lv}$, respectively. The existence of the magic numbers $Z=28$ and $N=50$ in those light fragments reinforces the strong influence of the magic structure of the heavy fragment.

The superasymmetric fission mode in SH nuclei discussed here differs from the asymmetric one observed in the Pu-Fm region, cf. Fig. 1(a). In the actinides, the heavy mass fragment is formed by the shell structure of the lighter doubly magic ${ }^{132} \mathrm{Sn}$ that produces a peak in the mass yield at $A_{H}=140$ [23]. The distinction between asymmetric and superasymmetric fission not only concerns the numerical values of most probable fragment masses. Qualitatively different shapes of the nucleus can be determined before scission in both modes. In $\mathrm{CR}$, the neck is short and narrow whereas in asymmetric fission in the actinides it is much longer and thicker [23]. As neck nucleons are shared between fragments at scission [37] the fragment mass yield in CR are expected to be much narrower than in asymmetric fission. The same conclusion may be deduced from the fact that the CR fission valley is very narrow in comparison with the asymmetric one. The variety of available fission shapes at the scission line is substantially reduced [38].

The same mechanism invoked here for spontaneous fission applies also to fusion-fission and quasifission observed in this region of SH nuclei [39-43]. In all the reactions the possible fragment mass asymmetry is forced by the shell structure of ${ }^{208} \mathrm{~Pb}$.

The predicted fission fragments produced by $\mathrm{CR}$ in $\mathrm{SH}$ nuclei lay out of the region of the fission products in the actinides, i.e., $A=60-180$ with maxima at $A_{L} \approx 100$ and $A_{H} \approx 140$. The range of possible masses of fragments should be extended in experiments to find signatures of the kind of asymmetric fission. Nowadays, experimental techniques do not allow the identification of fission fragments in the $\mathrm{SH}$ region. We hope that in the near future it will be possible to determine also masses of the fragments to disentangle the dominant fission mechanism in these regions. From our theoretical analysis we expect that the heavy fragment mass yield should be concentrated around $A=208$.

Acknowledgments. This work was partly supported by the Polish National Science Centre under Contracts No. 2016/21/B/ST2/01227 and No. 2017/24/T/ST2/00396. The work of L.M.R. was supported by Spanish Grants No. FPA2015-65929-P MINECO and No. FIS2015-63770-P MINECO.
[1] H. Rose and G. Jones, Nature 307, 245 (1984).

[2] R. Bonetti and A. Guglielmetti, in Heavy Elements and Related
New Phenomena, edited by W. Greiner and R. K. Gupta (World Scientific, Singapore, 1999), Vol. 2, p. 643. 
[3] D. N. Poenaru, Y. Nagame, R. A. Gherghescu, and W. Greiner, Phys. Rev. C 65, 054308 (2002).

[4] A. Zdeb, M. Warda, and K. Pomorski, Phys. Rev. C 87, 024308 (2013).

[5] O. A. P. Tavares and E. L. Medeiros, Phys. Scr. 86, 015201 (2012).

[6] D. N. Poenaru, R. A. Gherghescu, and W. Greiner, Phys. Rev. C 83, 014601 (2011).

[7] D. N. Poenaru, R. A. Gherghescu, and W. Greiner, J. Phys. G: Nucl. Part. Phys. 39, 015105 (2012).

[8] Z. Ren, C. Xu, and Z. Wang, Phys. Rev. C 70, 034304 (2004).

[9] C. Qi, F. R. Xu, R. J. Liotta, and R. Wyss, Phys. Rev. Lett. 103, 072501 (2009).

[10] D. Ni, Z. Ren, T. Dong, and C. Xu, Phys. Rev. C 78, 044310 (2008).

[11] M. Balasubramaniam, S. Kumarasamy, N. Arunachalam, and R. K. Gupta, Phys. Rev. C 70, 017301 (2004).

[12] A. Sandulescu, D. Poenaru, and W. Greiner, Sov. J. Part. Nuclei 11, 528 (1980).

[13] H. J. Krappe and K. Pomorski, Theory of Nuclear Fission (Springer, Berlin, 2012).

[14] N. Schunck and L. M. Robledo, Rep. Prog. Phys. 79, 116301 (2016).

[15] M. Warda and L. M. Robledo, Phys. Rev. C 84, 044608 (2011).

[16] D. N. Poenaru, R. A. Gherghescu, and W. Greiner, Phys. Rev. Lett. 107, 062503 (2011).

[17] D. N. Poenaru, R. A. Gherghescu, and W. Greiner, Phys. Rev. C 85, 034615 (2012).

[18] Y. L. Zhang and Y. Z. Wang, Phys. Rev. C 97, 014318 (2018).

[19] B. K. P. Santhosh, Nucl. Phys. A 929, 20 (2014).

[20] W. Nazarewicz, Nat. Phys. 14, 537 (2018).

[21] G. Martínez-Pinedo, D. Mocelj, N. Zinner, A. Kelić, K. Langanke, I. Panov, B. Pfeiffer, T. Rauscher, K.-H. Schmidt, and F.-K. Thielemann, Prog. Part. Nucl. Phys. 59, 199 (2007).

[22] L. Robledo and M. Warda, Int. J. Mod. Phys. E 17, 204 (2008).

[23] M. Warda, J. L. Egido, L. M. Robledo, and K. Pomorski, Phys. Rev. C 66, 014310 (2002).

[24] M. Warda and J. L. Egido, Phys. Rev. C 86, 014322 (2012).

[25] A. Baran, M. Kowal, P.-G. Reinhard, L. Robledo, A. Staszczak, and M. Warda, Nucl. Phys. A 944, 442 (2015).
[26] Y. Oganessian, J. Phys. G: Nucl. Part. Phys. 34, R165 (2007).

[27] C. E. Düllmann, M. Schädel, A. Yakushev, A. Türler, K. Eberhardt, J. V. Kratz, D. Ackermann, L.-L. Andersson, M. Block, W. Brüchle et al., Phys. Rev. Lett. 104, 252701 (2010).

[28] Y. T. Oganessian, Radiochim. Acta 99, 429 (2011).

[29] M. Wang, G. Audi, A. Wapstra, F. Kondev, M. MacCormick, X. Xu, and B. Pfeiffer, Chin. Phys. C 36, 1603 (2012).

[30] P. Möller, A. Sierk, T. Ichikawa, and H. Sagawa, At. Data Nucl. Data Tables 109-110, 1 (2016).

[31] H. Abusara, A. V. Afanasjev, and P. Ring, Phys. Rev. C 85, 024314 (2012).

[32] A. Staszczak, A. Baran, and W. Nazarewicz, Phys. Rev. C 87, 024320 (2013).

[33] A. Baran, Z. Łojewski, K. Sieja, and M. Kowal, Phys. Rev. C 72, 044310 (2005).

[34] P. Möller, A. J. Sierk, T. Ichikawa, A. Iwamoto, and M. Mumpower, Phys. Rev. C 91, 024310 (2015).

[35] P. Jachimowicz, M. Kowal, and J. Skalski, Phys. Rev. C 95, 014303 (2017).

[36] G. Audi, F. Kondev, M. Wang, B. Pfeiffer, X. Sun, J. Blachot, and M. MacCormick, Chin. Phys. C 36, 1157 (2012).

[37] M. Warda, A. Staszczak, and W. Nazarewicz, Phys. Rev. C 86, 024601 (2012).

[38] A. Zdeb, A. Dobrowolski, and M. Warda, Phys. Rev. C 95, 054608 (2017).

[39] E. M. Kozulin, G. N. Knyazheva, I. M. Itkis, M. G. Itkis, A. A. Bogachev, E. V. Chernysheva, L. Krupa, F. Hanappe, O. Dorvaux, L. Stuttgé et al., Phys. Rev. C 90, 054608 (2014).

[40] E. M. Kozulin, G. N. Knyazheva, K. V. Novikov, I. M. Itkis, M. G. Itkis, S. N. Dmitriev, Y. T. Oganessian, A. A. Bogachev, N. I. Kozulina, I. Harca et al., Phys. Rev. C 94, 054613 (2016).

[41] M. G. Itkis, I. M. Itkis, G. N. Knyazheva, and E. M. Kozulin, J. Phys.: Conf. Ser. 863, 012043 (2017).

[42] M. Itkis, J. Äystö, S. Beghini, A. Bogachev, L. Corradi, O. Dorvaux, A. Gadea, G. Giardina, F. Hanappe, I. Itkis et al., Nucl. Phys. A 734, 136 (2004).

[43] M. Itkis, A. Bogachev, I. Itkis, J. Kliman, G. Knyazheva, N. Kondratiev, E. Kozulin, L. Krupa, Y. Oganessian, I. Pokrovsky et al., Nucl. Phys. A 787, 150 (2007). 